\title{
Recent and new results on octonionic Bergman and Szeg \"o kernels
}

\author{
Rolf Soeren Krausshar ${ }^{1}$ \\ ${ }^{1}$ University of Erfurt
}

October 12, 2020

\begin{abstract}
Very recently one has started to study Bergman and Szeg $\backslash$ "o kernels in the setting of octonionic monogenic functions. In particular, explicit formulas for the Bergman kernel for the octonionic unit ball and for the octonionic right half-space as well as a formula for the Szeg \"o kernel for the octonionic unit ball have been established. In this paper we extend this line of investigation by developing explicit formulas for the Szeg “o kernel of strip domains of the form $\$\{\backslash$ cal $\{\mathrm{S}\}\}:=\backslash\{\mathrm{z} \backslash$ in $\backslash$ mathbb $\{\mathrm{O}\} \backslash$ mid $0<\backslash \operatorname{Re}(\mathrm{z})<\mathrm{d} \backslash\} \$$ from which we derive by a limit argument considering $\$ \mathrm{~d} \backslash$ to $\backslash$ infty $\$$ the Szeg $\backslash$ "o kernel of the octonionic right half-space. Additionally, we set up formulas for the Bergman kernel of such strip domains and relate both kernels with each other. In fact, these kernel functions can be expressed in terms of one-fold periodic octonionic monogenic generalizations of the cosecant function and the cotangent function, respectively.
\end{abstract}

\section{Hosted file}

KRAUSSHAR-octonionic Bergman1.pdf available at https://authorea.com/users/366427/articles/ 486199-recent-and-new-results-on-octonionic-bergman-and-szeg-o-kernels 